\title{
Förderung der Handlungsfähigkeit von Auszubildenden für die betriebliche Klimaanpassung
}

\author{
Christina Trautmann ${ }^{1}$ (D) - Alexander Siegmund ${ }^{1}$ \\ Eingegangen: 4. März 2021 / Überarbeitet: 14. September 2021 / Angenommen: 14. September 2021 / Online publiziert: 8. Oktober 2021 \\ (c) Der/die Autor(en) 2021
}

\section{Zusammenfassung}

Die regionalen Folgen des globalen Klimawandels sind längst auch in Deutschland unverkennbar. Die Temperaturen sind hier seit 1881 mit $1,6^{\circ} \mathrm{C}$ sogar stärker gestiegen als im globalen Vergleich, mit vielfältigen direkten und indirekten Auswirkungen für Umwelt, Gesellschaft und auch für die Wirtschaft. Die damit einhergehenden zunehmenden Extremwetterereignisse bergen vielfältige wirtschaftliche Risiken für Unternehmen und rücken die betriebliche Klimaanpassung damit in den Mittelpunkt des Interesses. Nur durch nachhaltige Anpassungsstrategien an die regional- und branchenspezifischen Folgen des Klimawandels können der wirtschaftliche Erfolg und die Wettbewerbsfähigkeit langfristig gesichert werden. Hierzu bedarf es jedoch eines sensibilisierten Bewusstseins sowie einschlägiger Kompetenzen zur Beurteilung der unternehmensspezifischen Vulnerabilität und zur Identifikation adäquater Anpassungsmaßnahmen. Im Rahmen des Vorhabens Transfer Together: Climate Change Education (TT:CCE) als Anschlussvorhaben von KlimAzubi wird die Zielgruppe der Auszubildenden von Unternehmen als die zukünftigen betrieblichen Akteure für die betriebliche Klimawandelbetroffenheit und Anpassung adressiert. Dies ist insbesondere in Anbetracht dessen wichtig, dass (inter-)nationale Studien einen geringen Bildungsstand von Jugendlichen und jungen Erwachsenen bezüglich der Strategien der Klimaanpassung aufzeigen.

Anhand des projektbezogenen Bildungsansatzes, der die Auswirkungen des Klimawandels auf die „,natürliche“ Umwelt vermittelt und gleichzeitig die Inhalte auf die „,betriebliche“ Umwelt transferiert, wird gezeigt, wie die betriebliche Klimaanpassung in der Berufsausbildung thematisiert und damit die (langfristige) Resilienz von Unternehmen gegenüber den Folgen des Klimawandels erhöht werden kann.

Schlüsselwörter Klimawandel $\cdot$ Bildungsprojekt $\cdot$ Klimabildung $\cdot$ Berufliche Bildung für Nachhaltige Entwicklung (BBNE) · Berufsausbildung

\begin{abstract}
The regional consequences of global climate change have long been undeniable in Germany as well. Since 1881, temperatures in Germany have increased even more than globally, by $1.6^{\circ} \mathrm{C}$, with multiple direct and indirect impacts on the environment, on society, and on the economy. The associated increase in extreme weather events poses a variety of economic risks for companies, and thus shifts corporate climate change adaptation to the centre of interest. Only through sustainable adaptation strategies addressing the regional- and sector-specific impacts of climate change long-term economic success and competitiveness can be ensured.
\end{abstract}

Christina Trautmann, M.Sc.

siegmund@ph-heidelberg.de

1 Abteilung Geographie - Research Group for Earth Observation (rgeo), UNESCO-Lehrstuhl für Erdbeobachtung und Geokommunikation an Weltwerbestätten und Biosphärenreservaten, Pädagogische Hochschule Heidelberg, Czernyring 22/10-12, 69115 Heidelberg, Deutschland 
However, this requires a sensitised awareness as well as relevant competencies for assessing a company's vulnerability to climate change and for identifying appropriate adaptation measures. Within the framework of the project Transfer Together: Climate Change Education (TT:CCE), the successor project of KlimAzubi, the target group of company trainees, as the future corporate actors for climate change impact and adaptation, is addressed. This is particularly important in view of the fact that (inter-)national studies show a low level of education in adolescents and young adults regarding climate change adaptation strategies.

Using the project-related educational approach, which conveys the effects of climate change on the 'natural' environment and, simultaneously transfers the contents to the 'business' environment, it is shown how operational climate change adaptation can be addressed in vocational training and thus increase the (long-term) resilience of companies to the impacts of climate change.

Keywords Climate change Educational project - Climate change education $\cdot$ Vocational education for sustainable development $\cdot$ Apprenticeship

\section{Einleitung}

Der Klimawandel ist nicht mehr nur eine abstrakte und ferne Gefahr, sondern eine globale und längst gegenwärtige Bedrohung, für die es zur Verhinderung fataler Schäden zeitnaher Anpassung bedarf. Dies gilt auch für ein auf den ersten Blick vermeintlich weniger betroffenes Land wie Deutschland. Denn während die mittlere Temperatur der Erde seit 1880 um knapp $1,1^{\circ} \mathrm{C}$ angestiegen ist (WMO 2020), wird für diese Periode in Deutschland ein Anstieg von bereits $1,6^{\circ} \mathrm{C}$ dokumentiert (UBA 2021). Dieser überdurchschnittliche Temperaturanstieg und der damit einhergehende Klimawandel führen zu vielfältigen Folgen für Umwelt, Gesellschaft und nicht zuletzt auch für die Wirtschaft (Siegmund 2008, Buth et al. 2017). Beispiele finden sich in allen Wirtschaftsbranchen: Hitze- und Trockenstress belasten die Forst- und Landwirtschaft, Hoch- und Niedrigwasser als Folge erschweren die Schifffahrt, Schäden an Straßen und Schieneninfrastrukturen durch starke Temperaturschwankungen beeinträchtigen Logistik und Verkehr, die Energieverbräuche und damit die Kosten für Kühlinfrastrukturen steigen und Elementarschäden durch Extremwetterereignisse nehmen zu (Adelphi et al. 2015). Gerade auch die jüngsten Starkniederschläge und das anschließende Hochwasser im Ahrtal in Westdeutschland, aber auch die Hitzewelle und ausgedehnte Waldbrände im Mittelmeerraum zeigen immer deutlicher die verstärkten Wetterextreme, mit denen zu rechnen ist.

Bleibt der globale Klimaschutz weiterhin wenig erfolgreich, werden viele bereits gegenwärtige Folgen des Klimawandels zukünftig intensiver und häufiger auftreten und weitere dazu kommen (IPCC 2021). Doch auch bei erfolgreichen Bemühungen, den globalen Temperaturanstieg auf das von den Mitgliedsstaaten der Vereinten Nationen beschlossene Ziel von unter $2{ }^{\circ} \mathrm{C}$ gegenüber dem vorindustriellen Niveau zu begrenzen (UN 2015), werden unumkehrbare Folgen bleiben. Der Weltklimarat (Intergovernmental Panel on Climate Change, IPCC) spricht sich so- gar dafür aus, das Ziel auf $1,5^{\circ} \mathrm{C}$ zu begrenzen, um essenzielle Kipppunkte im Klimasystem nicht zu überschreiten (Hoegh-Guldberg et al. 2018). Der Strategie der Klimaanpassung kommt somit eine unverkennbare Rolle zu, auch im betrieblichen Umfeld.

Für Unternehmen sind sowohl natürlich-physische (z.B. Hitzewellen) als auch regulative (z. B. Emissionsbepreisungen) und marktwirtschaftliche (z.B. veränderte Produktnachfragen) Klimawandelfolgen als Risiken zu identifizieren (Adelphi et al. 2015). Diese sind dabei angesichts der engen globalen Wirtschaftsvernetzung und dem damit verbundenen internationalen Transfer entlang der Wertschöpfungsketten für das betriebliche Risikomanagement von großer Bedeutung (Benzie et al. 2016; Peter et al. 2020). Für die betriebliche Risikoeinschätzung gegenüber den Folgen des Klimawandels sowie für die Entwicklung nachhaltiger regional- und branchenspezifischer Anpassungsstrategien ist es somit wichtig, auch die Auswirkungen auf Produktion, Distribution und Markt entlang der Wertschöpfungsketten zu berücksichtigen (Mahammadzadeh et al. 2013; Levermann 2014).

Dabei sind Kenntnisse zu diesen klimabedingten Herausforderungen durch den langen Zeithorizont des Klimawandels nicht nur in den jeweiligen Führungs- und Managementebenen von Unternehmen notwendig, sondern erfordern auch bei den gegenwärtig jungen Generationen und damit zukünftig Agierenden ein sensibilisiertes Bewusstsein (Anderson 2012). Das vom BMBF (Bundesministerium für Bildung und Forschung) geförderte Vorhaben Transfer Together: Climate Change Education (TT:CCE) führt das vom BMU (Bundesministerium für Umwelt, Naturschutz und nukleare Sicherheit) geförderte Vorgängerprojekt KlimAzubi der Abteilung Geographie - Research Group for Earth Observation ( ${ }^{\mathrm{r}} \mathrm{geo}$ ) ebendort fort und sensibilisiert die Auszubildenden verschiedener Unternehmen in der Metropolregion Rhein-Neckar für die regional- und branchenspezifisch differenzierten Folgen des Klimawandels. Indem die Teilnehmenden die Auswirkungen des Klimawandels auf 
ihr Unternehmen untersuchen und im Rahmen mehrtägiger Workshops gemeinsam Handlungsoptionen entwickeln, werden Kompetenzen für den Umgang mit klimabedingten Risiken, Unsicherheiten und Anpassungsoptionen gefördert.

\section{Klimawandelverständnis von Jugendlichen und jungen Erwachsenen}

Der Shell-Jugendstudie aus dem Jahr 2019 zufolge nennen $65 \%$ der Jugendlichen in Deutschland im Alter von 12-25 Jahren den Klimawandel als die gegenwärtige Herausforderung, die ihnen am meisten Angst bereitet (Albert et al. 2019, S. 15). Die junge Generation ist sich der Klimakrise bewusst. Dies ist aus 2 Perspektiven bedeutsam: als erste Generation wird sie die Auswirkungen des Klimawandels über die gesamte Spanne ihres Daseins erfahren und gleichzeitig eine fundamentale Rolle in der Bekämpfung des Klimawandels sowie in der Bewältigung zugehöriger Folgen einnehmen (Corner et al. 2015; Ojala und Lakew 2017). Beim Klimaschutz (Maßnahmen zur Minderung der Ursachen des Klimawandels, z. B. Reduzierung klimawirksamer $\mathrm{CO}_{2}$-Emissionen) als auch bei der Klimaanpassung (Maßnahmen zur Vermeidung oder Minderung der Folgen des Klimawandels, z.B. verbesserte Kühlinfrastrukturen) nehmen Jugendliche und junge Erwachsene somit eine zentrale Funktion als Akteure für die notwendige und bevorstehende soziale, ökonomische und ökologische Transformation ein (Nche et al. 2019).

Doch die Kenntnisse der jungen Generationen bezüglich der Ursachen und Folgen des Klimawandels sowie entsprechender Bewältigungsstrategien (Klimaschutz $=$ ursachenbezogen und Klimaanpassung $=$ folgenbezogen) sind zu Teilen immer noch von Missverständnissen geprägt, wie z.B. Verwechselungen mit allgemeinen Umweltschutzthemen (McNeill und Vaughn 2012; Lee et al. 2020). Insbesondere zur Klimaanpassung als Strategie zur Minderung der Empfindlichkeit natürlicher und menschlicher Systeme gegenüber den Folgen des Klimawandels zeigt eine aktuelle Studie von Graulich et al. (2020, S. 8-9), dass nur jeder vierte Jugendliche in Deutschland im Alter von 15-19 Jahren die Klimaanpassung korrekt definieren bzw. konkrete Beispiele nennen kann.

Der Aufbau einer resilienten (widerstandsfähigen) Gesellschaft und Wirtschaft gegenüber den Folgen des Klimawandels ist unabdingbar und die gegenwärtig junge Generation spielt dabei eine wichtige Rolle. Um den Klimawandel als ökologischen, ökonomischen und sozialen Risikofaktor wahrnehmen zu können, ist grundsätzliches Wissen zum Thema vonnöten (Lee et al. 2015), was die Notwendigkeit für Klimabildungsmaßnahmen mit Schwerpunkt auf der Klimaanpassung begründet.

\section{Climate Change Education - Klimaanpassung durch Auszubildende}

Die Wirksamkeit von Lernangeboten zur Klimabildung wird über die persönliche Relevanz des Themas für die Lernenden mitbestimmt. Je greifbarer und konkreter die Fragestellung, desto langfristiger der Erfolg der Maßnahme (Anderson 2012). Das Vorhaben TT:CCE der Abteilung Geographie - ${ }^{\mathrm{r} g e o}$ und dem dort angesiedelten Geco-Lab, Kompetenzzentrum für geoökologische Raumerkundung, an der Pädagogischen Hochschule Heidelberg führt das Projekt KlimAzubi fort und hat die Absicht, die Auszubildenden von Unternehmen als die zukünftigen betrieblichen Entscheidungsträger und Bottom-up-Innovatoren für die Vulnerabilität des eigenen Unternehmens gegenüber den Folgen des Klimawandels zu sensibilisieren. Der Fokus liegt dabei auf der Metropolregion Rhein-Neckar, eine bundeslandübergreifende und wirtschaftsstarke Region im süddeutschen Raum. Mit vielen (inter-)national agierenden Unternehmen kommt der betrieblichen Klimaanpassung hier eine wichtige Rolle zu. Zudem ist die Zielgruppe der Auszubildenden mit mehr als einer halben Million jährlich neu abgeschlossenen Ausbildungsverträgen in Deutschland (BMBF 2019, S. 51) für die zukünftige Resilienz von Unternehmen gegenüber den Folgen des Klimawandels relevant. Im Rahmen mehrtägiger Workshops (i.d.R. 3-4 Tage) wird bei den Auszubildenden die persönliche Betroffenheit sowie die betriebliche Vulnerabilität gegenüber den Folgen des Klimawandels thematisiert und entsprechende Gestaltungs- und Handlungsmöglichkeiten für eine nachhaltige Klimaanpassung verdeutlicht. Dabei werden verschiedene betriebliche Akteure wie z.B. Ausbilder/innen, HR-Manager/innen oder Geschäftsführer/innen in der Planung der Bildungskonzepte berücksichtigt, sodass das Thema mehrperspektivisch Beachtung findet. So wird das notwendige Bewusstsein der Auszubildenden bei der Entwicklung von nachhaltigen Lösungsansätzen zum Klimawandel gefördert, da diese aus verschiedenen Arbeitsbereichen kommen und damit auf unterschiedliche Weise von Klimawandelfolgen betroffen sein können. Den Kern des Projekts bildet der im Vorgängerprojekt entwickelte methodisch-didaktische Dreiklang, der die Folgen des Klimawandels für die „,natürliche“ mit denen für die „betriebliche“ Umwelt verknüpft (Abb. 1).

Um den Auszubildenden die klimawandelbedingte Vulnerabilität und die damit verbundenen Risiken und Chancen für die jeweilige Wirtschaftsbranche näherzubringen, sieht der Dreiklang eine mehrstufige Modulabfolge vor Erkennen, Analysieren und Beurteilen von Klimawandelfolgen auf die „natürliche“ Umwelt bzw. die „betriebliche“ Umwelt.

Durch die Betrachtung der „natürlichen“ Umwelt wird eine Wissensgrundlage geschaffen, die das Erkennen der 


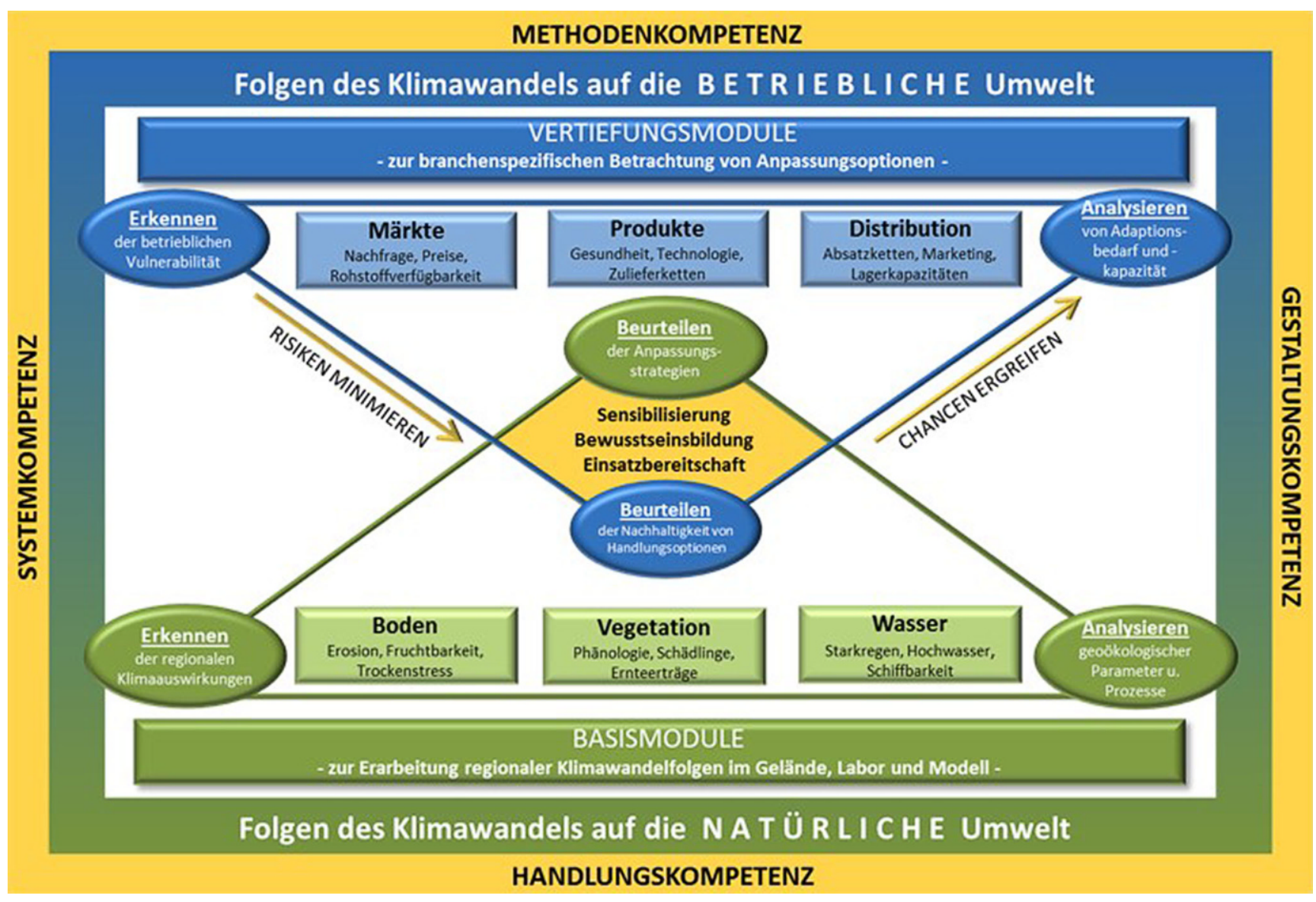

Abb. 1 Konzeptioneller Aufbau des doppelten methodisch-didaktischen Dreiklangs, der im Rahmen des KlimAzubi-Projekts entwickelt und evaluiert wurde und in TT:CCE Anwendung findet. (Quelle: $\left.{ }^{\mathrm{r}} \mathrm{geo}\right)$

Folgen des Klimawandels im Gelände und die vertiefende Analyse klimarelevanter Prozesse im Labor mit Bezug zu den natürlichen Ressourcen Boden, Vegetation und Wasser ermöglicht. Hierauf erfolgt die Beurteilung des Klimawandelrisikos für die „natürliche“ Umwelt sowie der Wirksamkeit und Nachhaltigkeit von Anpassungsmaßnahmen. Digitale Tools, z.B. das Portal www.klimafolgenonline. com des Potsdam-Instituts für Klimafolgenforschung (PIK) e. V. und der WetterOnline Meteorologische Dienstleistungen $\mathrm{GmbH}$, ermöglichen zudem eigenständige Untersuchungen zu den gegenwärtigen und zukünftigen regional differenzierten Folgen des Klimawandels unter verschiedenen Emissionsszenarien. So wird das Bewusstsein für die mehrdimensionalen (ökologischen, ökonomischen, sozialen) Folgen des Klimawandels gefördert, das als Basis für die Reflexion und den Transfer entsprechender Lerninhalte auf die „,betriebliche“ Umwelt dient (Abb. 2).

Im Rahmen der „betrieblichen“ Umwelt werden die Perspektiven der Vulnerabilität und Resilienz beleuchtet und so das Erkennen der betrieblichen Betroffenheit und das Analysieren von Anpassungsbedarf und -kapazität mit Relevanz für Märkte, Produktion und Distribution gefördert. Der
Schritt der Beurteilung bezieht sich dabei auf die Nachhaltigkeit verschiedener Handlungsoptionen zur Klimaanpassung. Hierfür kommen die Methoden der Szenariotechnik und des Design Thinkings in den Workshops zum Einsatz. Bei ersterer handelt es sich um eine mehrphasige Methode mittels derer die Zukunft unter Berücksichtigung verschiedener Entwicklungspfade (Szenarien) verständlich skizziert werden kann (Weinbrenner 2001; Reich 2010). Der erste Schritt der (1) Problemanalyse als Grundlage für die Szenariotechnik wird in den Lerneinheiten im Gelände und Labor sowie am digitalen Modell bereits abgehandelt. Im Rahmen der (2) Einflussanalyse werden unter Bereitstellung von Informationsmaterialien im Brainstorming-Verfahren für das Unternehmen relevante Probleme (natürlich-physisch, regulativ, marktwirtschaftlich) durch den Klimawandel gesammelt. In der (3) Deskriptorenanalyse werden diese Probleme hinsichtlich der Beeinflussbarkeit durch das Unternehmen analysiert und mögliche Messgrößen zur Abschätzung der Risiken definiert. Auf Basis dessen werden Best-Case- und Worst-Case-Szenarien skizziert (Phase 4), die die alternativen Entwicklungsmöglichkeiten des Unternehmens unter veränderten Klimabedingungen aufzeigen. Die Phase der 

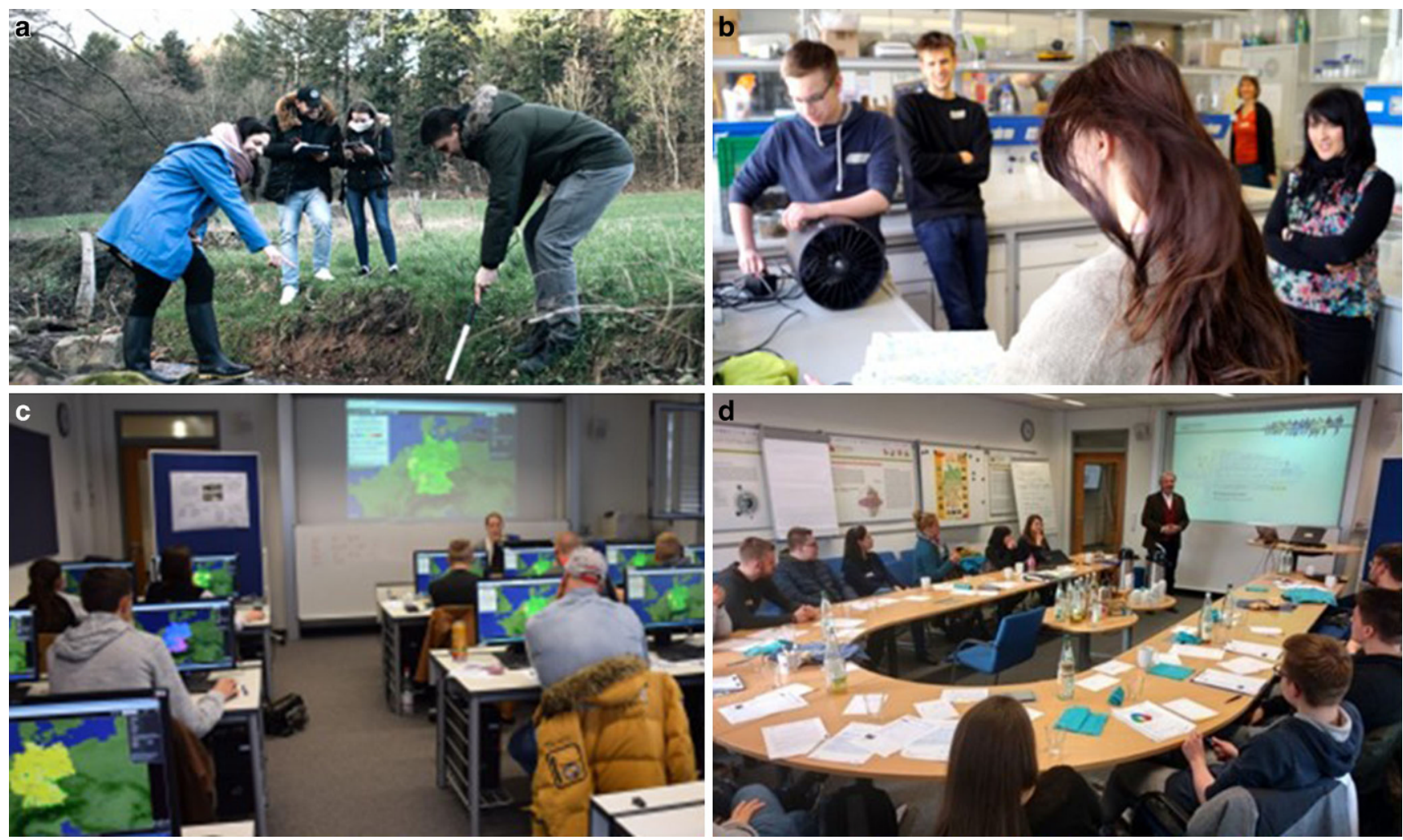

Abb. 2 Lernorte und -formate für „,natürlich““ und „betriebliche“ Umwelt im Rahmen des CCE-Projekts. (a) Geländeerhebungen, (b) Experimente und Modelle, (c) Computersimulationen, (d) Szenariotechnik \& Design Thinking. (Quelle: rgeo)

(5) Entwicklung von Strategien und Maßnahmen zur Problemlösung wird im Design Thinking umgesetzt.

Als kreativitätsförderndes Lernsetting erlaubt das Design Thinking die Genese problemzentrierter Innovationen im Kontext der Klimaanpassung (Lütke 2020). Die Befriedigung menschlicher Bedürfnisse steht dabei unter Berücksichtigung von Wirtschaftlichkeit und Umsetzbarkeit im Mittelpunkt (Grots und Pratschke 2009). Im Rahmen der Workshops werden durch die Methode nachhaltige Anpassungsoptionen für spezifische Unternehmensbereiche (z. B. relevante Beschaffungs- und Absatzmärkte), Produkte (z. B. Abschätzung der zukünftigen Nachfrage) und deren Distribution (z. B. Kühlketten) erarbeitet. Dabei wird das traditionelle Verständnis von Klimaanpassung als technikbasierte Strategie (Klein 2011) um temporäre, leitende und strategische Ansätze erweitert. So wird beispielsweise die Frage gestellt, ob der klimawandelbedingten sommerlichen Hitzebelastung mit dem Einsatz von Klimaanlagen als technische Lösung begegnet werden muss, oder ob durch organisatorische (leitende) Maßnahmen wie die Anpassung der Kernarbeitszeiten ähnliche Effekte erzielt werden können.

\section{Projektkontext und adressierte Branchen}

Das Projekt TT:CCE greift als Folgeprojekt des vom Umweltbundesamt mit dem Goldenen Kompass 2018 ausgezeichneten Projekts KlimAzubi der Abteilung Geographie ${ }^{r}$ geo der Pädagogischen Hochschule Heidelberg die Klimawandelbildung für die Zielgruppe der betrieblichen Auszubildenden auf. Im Rahmen von KlimAzubi wurden in Kooperation mit der Industrie- und Handelskammer (IHK) Rhein-Neckar und mit ausgewählten Unternehmen unterschiedlicher Wirtschaftsbranchen innovative Lernmodule zur betrieblichen Klimaanpassung entwickelt, evaluiert und disseminiert. Für die Auszubildenden der Rewe GmbH (Groß- und Einzelhandel), der HeidelbergCement AG (Baustoffbranche), der ABB Ltd. (Verarbeitendes Gewerbe) wurden, aufbauend auf den für die „natürliche“ Umwelt zentralen Themenfeldern Boden, Vegetation und Wasser, weitere branchenrelevante Klimawandelfolgen mit Auswirkung auf die Wirtschaft aufgegriffen:

Menschliche Gesundheit: Neben den physischen Gefährdungen durch Extremwetterereignisse, wie z. B. Stürme, sowie der Zunahme von Allergenen und vektorübertragenen Infektionskrankheiten wird insbesondere der Umgang mit Hitzebelastung am Arbeitsplatz unter den Vorzeichen der zu erwartenden Temperaturanstiege bedeutsam. 
Binnenschifffahrt: Hochwasser beeinträchtigt die Unternehmenslogistik durch überflutete Verkehrswege und Nichtschiffbarkeit der Wasserstraßen. Mit zunehmenden winterlichen Niederschlagssummen wird sich die Hochwasserproblematik in Zukunft verstärken. Aber auch die zurückgehenden Sommerniederschläge, die häufiger nach längeren trocken-heißen Phasen als vereinzelte Starkniederschlagsereignisse auf wenig wasseraufnahmefähige Böden treffen, können Hochwasser verursachen.

Landwirtschaft: Insbesondere für die lebensmittelverarbeitenden Branchen sowie den Groß- und Einzelhandel sind die Verfügbarkeiten der landwirtschaftlich erzeugten Nahrungsmittel zentral. Extremereignisse wie Hagel oder heiße Trockenphasen gefährden die Erntemenge und -qualität. Hitze erschwert zudem den Transport und die Lagerung leicht verderblicher Lebensmittel.

Verkehr und Verkehrsinfrastruktur: Neben den bereits genannten hoch- bzw. niedrigwasserbedingten Unterbrechungen der Verkehrswege sind auch die Schäden durch Sturm (z.B. Oberleitungsschäden) oder Hitze (z. B. Blow-ups auf Autobahnen) wahrscheinlich.

Zur dauerhaften Verankerung und Förderung der weiteren Dissemination der Bildungsmodule wurde den im KlimAzubi-Projekt kooperierenden Unternehmen nach den Workshops ein individueller Methodenkoffer ausgehändigt. Auch bei der IHK Rhein-Neckar wurde ein solcher Koffer hinterlegt, der kostenfrei ausgeliehen werden kann. Im Folgeprojekt TT:CCE werden die Lernmodule auf weitere Branchen transferiert, z. B. auf die Containerlogistik (Contargo GmbH \& Co. KG), die Nahrungsmittelbranche (Südzucker AG) (Nahrungsmittel) und den Energiesektor (Mvv Energie AG). Bisher wurden durch beide Projekte insgesamt mehr als 350 Auszubildende erreicht.

\section{Beispiel: Klimarisiken im Verarbeitenden Gewerbe}

Das Verarbeitende Gewerbe ist meist eng in die internationale Wirtschaft eingebunden und damit ist die Abhängigkeit von Zulieferern und Abnehmern hoch. Natürlich-physische (I), regulative (II) sowie marktwirtschaftliche (III) Klimawandelfolgen spielen eine wichtige Rolle. Folgende Faktoren wurden im Rahmen der Workshops für das Verarbeitende Gewerbe (bisher) thematisiert:

- Hitzebelastung (I): Hochtechnisierte Produktionsprozesse erfordern konstante Temperaturen. Mit zunehmender Hitzebelastung im Sommer kann damit die Produktion direkt beeinflusst werden und auch eine Belastung für die Mitarbeiter mit Konzentrations- und Leistungs- fähigkeitseinbußen darstellen (Hellwig et al. 2012). Im Rahmen der Workshops werden nachhaltige Kühlungsmöglichkeiten zur Prävention hitzebedingter Zwischenfälle im Betriebsablauf anhand von Temperaturmessungen verschiedener Baustoffe bei unterschiedlichen Einstrahlungsbedingungen sowie Thermoexperimenten zur Reduktion der Innentemperaturen an einem Modellhaus (Labor) erarbeitet.

- Hoch- und Niedrigwasser (I): Hoch- und Niedrigwasser bei bedeutsamen Binnenwasserstraßen als Folge von Starkniederschlägen bzw. intensiven Trockenperioden können sich negativ auf Zuliefer- und Absatzketten auswirken. Dies ist insbesondere in Anbetracht des eng verknüpften Warenaustauschs durch arbeitsgeteilte Wertschöpfung und der beliebten Just-in-time-Produktion relevant (Ott und Richter 2008). Denn mit geringen Lagerkapazitäten sinkt die Resilienz der Produktion gegenüber Verspätungen von Zulieferern und Abnehmern. Im Kontext der „natürlichen“ Umwelt führen die Auszubildenden Messungen von Fließgeschwindigkeit und Fließverhalten im Gelände durch und kartieren die Gewässerstrukturgüte an naturnahen und naturfernen Gewässern. Hierdurch werden die Fertigkeiten zur Einschätzung lokaler Hochwasserrisiken vermittelt. Mit entsprechenden Modellen im Labor werden im nächsten Schritt die natürlichen (z. B. Starkniederschläge) und anthropogenen (z. B. Flächenversiegelung) Faktoren für die Entstehung von Hochwasser erläutert und der Transfer auf die „betriebliche“ Umwelt anhand von Beispielen, z.B. produktspezifischen Lieferanten- und Kundennetzwerken, gefördert.

- Energieeffizienzanforderungen (II): Die nationalen Beiträge zum Erreichen des globalen Klimaschutzziels sind häufig von neuen Verordnungen zur Energieeinsparung und Energieeffizienz begleitet. Im betrieblichen Kontext können davon sowohl die energetischen Rahmenbedingungen für die Produktion als auch die Anforderungen an die hergestellten Produkte (z. B. Elektrogeräte) betroffen sein. Je nach räumlicher Ausprägung des Absatzmarkts (national/international) sind regulative Klimawandelfolgen für den zukünftigen wirtschaftlichen Erfolg eines Unternehmens von großer Bedeutung. Mithilfe der Szenariotechnik werden den Teilnehmenden die unterschiedlichen betrieblichen Negativentwicklungen durch mangelnde Klimaanpassung aufgezeigt.

- Änderungen der Nachfrage (III): Die Nachfrage nach einem Produkt wird von vielen Faktoren mitbestimmt. Neben Nutzen und Qualität spielt auch der Preis eine wichtige Rolle. Die Rentabilität eines Produkts in einer vom Klimawandel geprägten Zukunft muss abgewogen werden. Zudem kann der Produktpreis durch erhöhte Rohstoffpreise und durch die Einführung regulativer Maßnahmen zum Klimaschutz, z.B. einer $\mathrm{CO}_{2}$-Steuer, 
stark erhöht werden und damit die Nachfrage reduzieren (Peter et al. 2019). Die Szenariotechnik erlaubt es, auch die meist weniger greifbaren Folgen des Klimawandels $\mathrm{zu}$ thematisieren und bei den Teilnehmenden damit eine Grundlage für die betriebliche Risikoeinschätzung zu schaffen.

\section{Fazit und Ausblick}

Kenntnisse zur zukünftigen Resilienz von Unternehmen gegenüber den Folgen des Klimawandels und damit zur Klimaanpassung sind für die Wirtschaft essenziell, doch Jugendliche und junge Erwachsene als die Entscheidungsträger von morgen wissen wenig darüber. Das Projekt KlimAzubi hat exemplarisch gezeigt, wie in der beruflichen Bildung die betriebliche Klimawandelvulnerabilität und Klimaanpassung geschult und mittels der Methode des Design Thinkings die Kreativität der Auszubildenden zur Findung von Anpassungsmaßnahmen erhöht werden kann (Lütke 2020). Durch das Vorhaben TT:CCE wird das Netzwerk von klimavulnerablen Unternehmen in der Metropolregion Rhein-Neckar nun um neue Kooperationspartner erweitert und branchenübergreifend geöffnet. Die Zahl der teilnehmenden Auszubildenden wird somit weiter erhöht. Doch durch die branchenspezifischen Kursinhalte und die Kooperation mit der IHK Rhein-Neckar hat das Projekt eine deutlich größere Reichweite, da die Inhalte auch in den regulären Ausbildungsbetrieb übernommen werden können. Das anhaltende Interesse an weiteren Workshops seitens bereits bestehender Kooperationspartner zeigt, dass sich nach Auffassung der Unternehmen substanzielle Lerneffekte bei den Auszubildenden einstellen, die den mit den Workshops verbundenen organisatorischen und zeitlichen Aufwand rechtfertigen.

Erfahrungen aus beiden Projekten zeigen, dass die $\mathrm{Zu}$ sammenarbeit verschiedener Abteilungen in den Unternehmen sowohl in Vorbereitung als auch Durchführung der Bildungskonzepte die Sensibilisierung für die Thematik stärkt. Durch Absprachen mit verschiedenen Akteuren aus den Unternehmen wird die Wertschätzung der Ergebnisse seitens des Unternehmens erhöht und gemeinschaftlich erarbeitete Anpassungskonzepte entwickelt. So bekamen die Auszubildenden im Anschluss an den Workshop teilweise die Möglichkeit, ihre Anpassungsansätze in den Leitungsebenen des Unternehmens vorzustellen. Damit wird die Grundidee des Bildungsprojekts, über Auszubildende als Bottom-up-Innovatoren Ansätze zur betrieblichen Klimaanpassung in den Unternehmen zu etablieren, umfassend berücksichtigt und eine Grundlage für die langfristige Verankerung auf verschiedenen Unternehmensebenen geschaffen.
Funding Open Access funding enabled and organized by Projekt DEAL.

Open Access Dieser Artikel wird unter der Creative Commons Namensnennung 4.0 International Lizenz veröffentlicht, welche die Nutzung, Vervielfältigung, Bearbeitung, Verbreitung und Wiedergabe in jeglichem Medium und Format erlaubt, sofern Sie den/die ursprünglichen Autor(en) und die Quelle ordnungsgemäß nennen, einen Link zur Creative Commons Lizenz beifügen und angeben, ob Änderungen vorgenommen wurden.

Die in diesem Artikel enthaltenen Bilder und sonstiges Drittmaterial unterliegen ebenfalls der genannten Creative Commons Lizenz, sofern sich aus der Abbildungslegende nichts anderes ergibt. Sofern das betreffende Material nicht unter der genannten Creative Commons Lizenz steht und die betreffende Handlung nicht nach gesetzlichen Vorschriften erlaubt ist, ist für die oben aufgeführten Weiterverwendungen des Materials die Einwilligung des jeweiligen Rechteinhabers einzuholen.

Weitere Details zur Lizenz entnehmen Sie bitte der Lizenzinformation auf http://creativecommons.org/licenses/by/4.0/deed.de.

\section{Literatur}

Adelphi, PRC und EURAC (2015) Vulnerabilität Deutschlands gegenüber dem Klimawandel. Umweltbundesamt, Dessau-Roßlau

Albert M, Hurrelmann K, Quenzel G, Schneekloth U, Leven I, Wolfert S, Utzmann H (2019) Shell Jugendstudie. Shell. https://www. shell.de/ueber-uns/shell-jugendstudie/_jcr_content/par/toptasks. stream/1570708341213/

4a002dff58a7a9540cb9e83ee0a37a0ed8a0fd55/shell-youthstudy-summary-2019-de.pdf. Zugegriffen: 22. Febr. 2021

Anderson A (2012) Climate change education for mitigation and adaptation. UNESCO special section on the ESD response to the three Rio conventions. Journal of Education for Sustainable Development 6(2):191-206. https://doi.org/10.1177/0973408212475199

Benzie M, Hedlund J, Carlsen H (2016) Introducing the transnational climate impacts index: indicators of country-level exposure-Methodology report. Strockholm Environment Institute, Stockholm

BMBF (2019) Berufsbildungsbericht. Bundesministerium für Bildung und Forschung (BMBF) Referat Grundsatzfragen der beruflichen Aus- und Weiterbildung, Bonn

Buth M, Kahlenborn W, Greiving S, Fleischhauer M, Zebisch M, Schneiderbauer S, Schauser I (2017) Leitfaden für Klimawirkungs- und Vulnerabilitätsanalysen. Umweltbundesamt, DessauRoßlau

Corner A, Roberts O, Chiari S, Völler S, Mayrhuber ES, Mandl S, Monson K (2015) How do young people engage with climate change? The role of knowledge, values, message framing, and trusted communicators. WIREs Clim Chang 6(5):523-534. https://doi.org/10.1002/wcc.353

Graulich D, Schärling R, Kuthe A, Fiene C, Siegmund A (2020) Young people and their (mis)conceptions on climate change adaptation. In: Filho WL, Luetz JM, Ayal DY (Hrsg) Handbook of climate change management. Springer, Cham, S 1-19 https://doi.org/10. 1007/978-3-030-22759-3_202-1

Grots A, Pratschke M (2009) Design Thinking - Kreativität als Methode. Thexis 26:18-23. https://doi.org/10.1007/s11621-009-0027-4

Hellwig RT, Nöske I, Brasche S, Gebhardt HJ, Levchuk I, Bischof W (2012) Hitzebeanspruchung und Leistungsfähigkeit in Büroräumen bei erhöhten Außentemperaturen. Bundesanstalt für Arbeitsschutz und Arbeitsmedizin, Dortmund, Berlin, Dresden

Hoegh-Guldberg O, Jacob D, Taylor M, Bindi M, Brown S, Camilloni I, Diedhiou A, Djalante R, Ebi KL, Engelbrecht F, Guiot J, Hijoka Y, Mehrotra S, Payne A, Seneviratne SI, Thomas A, War- 
ren $\mathrm{R}$, Zhoue $\mathrm{G}$ (2018) Impacts off $1.5^{\circ} \mathrm{C}$ of global warming on natural and human system. In: Masson-Delmotte VP, Pörtner HO, Roberts D, Skea J, Shukla PR, Pirani A, MoufoumaOkia W, Péan C, Pidcock R, Connors S, Matthews JB, Chen Y, Zhou X, Gomis MI, Lonnoy E, Maycock T, Tignor M, Waterfield $\mathrm{T}$ (Hrsg) Global warming of $1.5^{\circ} \mathrm{C}$. An IPCC special report, S $175-311$

IPCC (2021) Summary for Policymakers. In: Masson-Delmotte V, Zhai P, Pirani A, Connors SL, Péan C, Berger S, Caud N, Chen Y, Goldfarb L, Gomis MI, Huang M, Leitzell K, Lonnoy E, Matthews JBR, Maycock TK, Waterfield T, Yelekci O, Yu R, Zhou B (Hrsg) Climate change 2021: the physical science basis. Contribution of working group I to the sixth assessment report of the intergovernmental panel on climate change

Klein RJT (2011) Adaptation to climate change: more than technology. In: Linkov I, Bridges TS (Hrsg) Climate: global change and local adaptation, NATO science for peace and security series C: environmental security. Springer, Dordrecht, S 157-168

Lee K, Gjersoe N, O'Neill S, Barnett J (2020) Youth perceptions of climate change: a narrative synthesis. WIREs Clim Chang 11(3):1-24. https://doi.org/10.1002/wcc.641

Lee TM, Markowitz EM, Howe PD, Ko CY, Leiserowitz AA (2015) Perdictors of public climate change awareness and risk perception around the world. Nature Clim Change 5:1014-1020. https://doi. org/10.1038/nclimate2728

Levermann A (2014) Climate economics: make supply chains climatesmart. Nature 506:27-29. https://doi.org/10.1038/506027a

Lütke C (2020) Design Thinking und die Veränderung von Kreativität - im Kontext betrieblicher Anpassung an den Klimawandel. Dissertation, Pädagogische Hochschule Heidelberg

Mahammadzadeh M, Chrischilles E, Biebeler H (2013) Klimaanpassung in Unternehmen und Kommunen. Betroffenheit, Verletztlichkeit und Anpassungsbedarf. Institut der deutschen Wirtschaft, Köln. https://www.iwkoeln.de/fileadmin/user_upload/Studien/ IW-Analysen/PDF/Bd._83_Klimaanpassung.pdf. Zugegriffen: 28. Jan. 2021
McNeill KL, Vaughn MH (2012) Urban high school students' critical science agency: conceptual understandings and environmental actions around climate change. Res Sci Educ 42(2):373-399

Nche GC, Achunike HC, Okoli AB (2019) From climate change victims to climate change actors: the role of eco-parenting in building mitigation and adaptation capcities in children. J Environ Educ 50(2):131-144. https://doi.org/10.1080/00958964.2018.1553839

Ojala M, Lakew Y (2017) Young people and climate change communication. Oxford Res Encycl Clim Sci. https://doi.org/10.1093/ acrefore/9780190228620.013.408

Ott HE, Richter C (2008) Anpassung an den Klimawandel - Risiken und Chancen für deutsche Unternehmen. Wuppertal Institut für Klima, Umwelt, Energie GmbH, Wuppertal

Peter M, Guyer M, Füssler J (2019) Folgen des globalen Klimawandels für Deutschland. Erster Teilbericht: Die Wirkungsketten in der Übersicht. Umweltbundesamt, Dessau-Roßlau

Peter M, Guyer M, Füssler J, Bednar-Friedl B, Knittel N, Bachner G, Schwarze R, von Unger M (2020) Folgen des Klimawandels für Deutschland. Abschlussbericht: Analysen und Politikempfehlungen. Umweltbundesamt, Dessau-Roßlau

Reich K (2010) Szenario-Methode. Methodenpool. http://methodenpool. uni-koeln.de. Zugegriffen: 17. Juni 2021

Siegmund A (2008) Der Klimawandel - eine aktuelle Bilanz globaler Prozesse und ihrer regionalen Folgen. uwf 16:3-11. https://doi. org/10.1007/s00550-008-0068-1

UBA (2021) Trends der Lufttemperatur. Umweltbundesamt. https:// www.umweltbundesamt.de/daten/klima/trends-der-lufttemperatur\# steigende-durchschnittstemperaturen-weltweit. Zugegriffen: 5 . Febr. 2021

UN (2015) Paris Agreement. United Nations, Paris

Weinbrenner P (2001) Szenariotechnik. https://www.sowi-online.de/ praxis/methode/szenariotechnik.html. Zugegriffen: 9. Febr. 2021

WMO (2020) WMO confirms 2019 as second hottest year on record. https://public.wmo.int/en/media/press-release/wmo-confirms2019-second-hottest-year-record. Zugegriffen: 22. Febr. 2021 